\title{
Prevalencia de diabetes mellitus en perros adultos con sobrepeso en Cuenca, Ecuador
}

\author{
Omar S. Andrade ${ }^{1}$ Emmanuel L. Galarza ${ }^{2}$, Jhonny A. Narváez, Manuel T. Pesántez C. ${ }^{1}$ \\ ${ }^{1}$ Facultad de Ciencias Agropecuarias, Universidad de Cuenca, Cuenca, Ecuador. \\ ${ }^{2}$ Facultad de Medicina Veterinaria, Universidad Católica de Cuenca, Cuenca, Ecuador.
}

Autor de correspondencia: omar.andrade@ucuenca.edu.ec

Fecha de recepción: 29 de abril 2017 - Fecha de aceptación: 15 de junio 2017

\begin{abstract}
RESUMEN
La diabetes mellitus (DM) es un trastorno crónico del metabolismo de los carbohidratos como consecuencia a una deficiencia relativa de insulina. El objetivo de esta investigación fue determinar la prevalencia de diabetes mellitus en perros mayores a 7 años con sobrepeso. Un total de 250 perros que presentaron síntomas asociados a la enfermedad (poliuria, polidipsia, polifagia, letargia) fueron analizados para determinar los niveles de glucosa en sangre mediante el uso de un glucómetro. El porcentaje de animales hipoglucémicos fue de $26.8 \%$; no se encontraron animales con niveles de glucosa que indiquen la presencia de la enfermedad. Los datos fueron analizados en un modelo lineal general mixto a través del PROC MIXED en el SAS vw 9.3 (2013), que utilizó la metodología de máxima verosimilitud restringida (REML) y procedimiento Satterth Wait para ajustar las medias de los grados de libertad, mediante el cual se determinó que no existe significancia entre la relación edad y niveles de glucemia (p>0.05). Sin embargo, los niveles de glucosa sí tienen correlación con la dieta y el tamaño de la raza. Los perros que consumieron alimento casero obtuvieron valores altos de glucosa, $67.66 \mathrm{mg} \mathrm{dl}^{-}$ ${ }^{1}$, pero dentro de los rangos normales de glucemia. De igual manera, lo obtuvieron las razas grandes $70.55 \mathrm{mg} \mathrm{dl}^{-1}$. Síntomas de poliuria y polidipsia se presentaron en todos los animales; la polifagia y letargia se presentó en el 1.2\% de animales. Se concluye que no existe prevalencia de diabetes mellitus en la población de perros estudiada en el cantón Cuenca, Ecuador.
\end{abstract}

Palabras clave: Diabetes mellitus canina, hiperglucemia.

\begin{abstract}
Diabetes mellitus (DM) is a chronic disorder of carbohydrate metabolism because of a relative insulin deficiency. The objective of this research was to determine the prevalence of diabetes mellitus in overweight dogs over 7 years old. The blood glucose levels using the glucometer were determined on a total of 250 dogs that presented symptoms associated with the disease (polyuria, polydipsia, polyphagia, lethargy). The percentage of hypoglycaemic animals was $26.8 \%$; no animals were found with glucose levels indicating the presence of the disease. The data were analysed in a mixed general linear model through PROC MIXED in the SAS vw 9.3 (2013), which used the methodology of restricted maximum likelihood (REML) and the Satterth Wait procedure to adjust the means of the degrees of freedom. The analysis revealed that no significance relation exists between age and blood glucose levels ( $>0.05$ ). However, glucose levels correlate with diet and breed size. The dogs that consumed homemade food presented high values of glucose, $67.66 \mathrm{mg} \mathrm{dl}^{-1}$, within the normal ranges of glycemia. Similarly, large races possessed on average a glucose level of $70.55 \mathrm{mg} \mathrm{dl}^{-1}$. Symptoms of polyuria and polydipsia were present in all animals; polyphagia and lethargy occurred in $1.2 \%$ of animals. It is concluded that there is no prevalence of diabetes mellitus in the studied dog population of Cuenca, Ecuador.
\end{abstract}

Keywords: Canine diabetes mellitus, hyperglycemia. 


\section{INTRODUCCIÓN}

La diabetes mellitus (DM), endocrinopatía común en perros, tiene como consecuencia una alteración del metabolismo de los carbohidratos debido a una deficiencia absoluta de la insulina. Animales que padecen esta enfermedad necesitan de por vida la aplicación exógena de insulina para controlarla (Bonagura \& Twedt, 2009). La deficiencia de insulina puede producirse por una pérdida progresiva de células pancreáticas, esta destrucción de células es actualmente desconocida, pero se cree que esta destrucción puede estar asociada a procesos de algunas enfermedades, como las patologías endocrinas del páncreas, la destrucción inmunomediada, y la hipoplasia congénita de las células (Fall et al., 2007). Esta deficiencia de insulina también se puede dar por resistencia al efecto de la insulina causada por el antagonismo con otras hormonas, por ejemplo, en canes hembras al estar en etapa de diestro, o por otros desórdenes endocrinos como el hiperadrenocortisismo o la acromegalia (Catchpole et al., 2005). La diabetes tipo 1 parece ser la forma más común de diabetes en perros, y se caracteriza por la destrucción pancreática de células B que conduce a una deficiencia absoluta de insulina (Rand et al., 2004). Según Guptill et al. (2003), la edad es un factor predisponente para esta enfermedad, más común entre perros de 5 a 12 años y menos frecuente en animales menores de 3 años. La obesidad es otro factor para la aparición de la enfermedad (Catchpole et al., 2005). Davidson (2005) y Catchpole et al. (2005), al estudiar los factores predisponentes para que se presente la enfermedad en perros, concluyeron que la raza es un factor genético susceptible para desarrollar diabetes. Entre las razas señaladas están el Fox Terrier, Collie, Boxer, Schnauzer, y Pastor Alemán. La reducción de utilización de glucosa, aminoácidos y ácidos grasos tiene una manifestación clínica semiológica con: letargia, pérdida de peso, menor estimulación del centro de la saciedad, pelo hirsuto y disminución de las defensas orgánicas, características típicas de los perros diabéticos que no están en tratamiento (Fleeman \& Rand, 2006). El diagnóstico de la diabetes mellitus es sencillo y se basa en hallazgos clínicos como el aumento de la frecuencia en la micción ya que la glucosa actúa como un diurético osmótico que ocasiona: un aumento en la frecuencia de ingestión de líquidos para compensar la deshidratación, el catabolismo continuado a nivel muscular y de las grasas, así como las pérdidas calóricas en la orina que conducen a una polifagia y pérdida de peso (Hardy, 1998). La producción de glucosuria y el aumento de glucosa en la sangre, factores asociados a concentraciones de glucosa mayores a $180 \mathrm{mg} \mathrm{dl}^{-1}$, se pueden determinar mediante pruebas de laboratorio. Estas pruebas son automatizadas y utilizan métodos de hexoquinasa o glucosa oxidasa en plasma heparinizado. Pruebas de fructosamina son también usadas, comúnmente en perros y gatos, para monitorear los niveles de glucosa (Wiedmeyer \& Declue, 2011). Sin embargo, estos métodos de laboratorio necesitan grandes volúmenes de muestras y son laboriosos y costosos. Existen en la actualidad medidores portátiles de glucosa (glucómetros) en la sangre, útiles para mejorar el control glicémico en animales pequeños, son fáciles de usar, requieren pequeños volúmenes de sangre, son menos estresantes y proporcionan resultados rápidos (Kang et al., 2016). El tiempo aproximado de supervivencia de los perros con DM es de 3 años a partir del diagnóstico; la mayor mortalidad ocurre durante los primeros 6 meses (Fall et al., 2007). Poco se conoce sobre la epidemiología de esta enfermedad en perros del cantón Cuenca, de allí la importancia de esta investigación que pretende determinar la prevalencia de la diabetes mellitus en perros mayores de 7 años, que presenten una condición corporal de obesidad. Además, este estudio evalúa si existe correlación con las variables: edad, condición corporal, alimentación y raza.

\section{MATERIALES Y MÉTODOS}

\subsection{Selección de animales}

Este estudio fue realizado en el periodo de junio a diciembre del año 2015 en 10 clínicas veterinarias de la ciudad de Cuenca, Ecuador, en 250 perros de 25 razas que se agruparon en razas pequeñas, medianas y grandes. Los canes evaluados fueron hembras y machos adultos, con una edad igual o mayor a siete años y con una condición corporal de sobrepeso u obesidad. Para la valoración de la condición corporal se consideró la cantidad de grasa presente en el cuerpo del animal. Esta evaluación fue visual. Se asignó un valor de 4 (sobrepeso) para casos en los que fue difícil tocar las costillas y existía presencia de 
depósitos de grasa sobre el área lumbar y la base de la cola; valores de 5 (obeso) se asignaron a los casos en los que las costillas no pudieron ser palpadas, cintura difícilmente identificable, y presencia de abdomen redondeado y de grasa a lo largo de toda la columna vertebral (Laflamme, 1997). A cada animal se le realizó un examen clínico general para descartar la presencia de otras enfermedades e identificar síntomas relacionados con diabetes. El tipo de alimentación que recibían los animales, fue categorizado en: balanceado, alimento casero y mixto (mezcla de los dos).

\subsection{Toma de las muestras de sangre y medición de los niveles de glucosa}

La extracción de sangre se realizó mediante una punción en la arteria auricular, previa desinfección del área. Para la toma de muestras, los animales tuvieron un ayuno de al menos 8 horas. Una vez realizada la punción se impregnó una gota de sangre en la tira reactiva, la cual se introdujo en el glucómetro por 15 segundos antes de proceder a realizar la lectura de los niveles de glucosa en $\mathrm{mg} \mathrm{dl}^{-1}$. Los niveles obtenidos se compararon con los valores normales de 60 a $120 \mathrm{mg} \mathrm{dl}^{-1}$, referidos por Cunningham \& Klein (2009). Los animales que presentaron niveles inferiores a $60 \mathrm{mg} \mathrm{dl}^{-1} \mathrm{de}$ glucosa fueron clasificados como hipoglucémicos, aquellos que tuvieron rangos entre 60 y $120 \mathrm{mg} \mathrm{dl}^{-1}$ fueron considerados como normoglucémicos. No se encontraron perros con valores sobre $180 \mathrm{mg} \mathrm{dl}^{-1}$ (hiperglucémicos). La tira reactiva y el glucómetro fueron de la marca AccuChek® (Roche Inc.).

\subsection{Diagnóstico de diabetes mellitus}

Animales hiperglucémicos, con valores de glucosa superiores a $180 \mathrm{mg} \mathrm{dl}^{-1}$, en periodo de ayunas y que presenten síntomas como poliuria, polidipsia, polifagia y letargia se considerarán afectados de diabetes mellitus.

\subsection{Análisis estadístico}

Se realizó un estudio observacional, los datos fueron analizados en un modelo lineal general mixto a través del PROC MIXED en el SAS vw 9.3 (2013). Se utilizó la metodología de máxima verosimilitud restringida (REML) y el PROC SATTERTHWAITE (DDFM=SATTERTH) para obtener los grados de libertad correctos en la tabla de prueba de efectos fijos de las medias mínimo-cuadráticas calculadas.

\section{RESULTADOS}

\subsection{Niveles de glucosa}

Los perros con niveles normales de glucosa fueron los de mayor frecuencia (Tabla 1), seguidos por los animales hipoglucémicos. No se encontraron perros con niveles de hiperglucemia para relacionarlos con la sintomatología clínica de diabetes mellitus. La Tabla 1 indica los valores medios de los niveles de glucosa encontrados en los perros del estudio, distribuidos en perros con niveles normales (normoglucémicos), niveles bajos (hipoglucémicos) y niveles altos (hiperglucémicos) con el número de perros correspondientes a cada categoría.

Tabla 1. Niveles medios de glucemia en perros adultos ( $>7$ años) y con condición corporal mayor o igual a 4 (sobrepeso u obesidad) en la ciudad de Cuenca, Ecuador.

\begin{tabular}{lccc}
\hline Niveles de glucemia & $\mathrm{N}$ & $\bar{X} \mathrm{mg} \mathrm{dl}^{-1}$ & Porcentaje \\
\hline Normoglucémicos & 183 & 75 & 73.2 \\
Hipoglucémicos & 67 & 55 & 26.8 \\
Hiperglucémicos & 0 & 0 & 0 \\
\hline TOTAL & 250 & & 100 \\
\hline
\end{tabular}




\subsection{Relación de los niveles de glucosa según la edad, dieta y raza}

Se determinó que no existe asociación ( $p>0.05$ ) entre los factores edad y niveles de glucosa. En cambio, en los niveles de glucemia según la dieta, sí se determinaron diferencias altamente significativas ( $\mathrm{p}<0.01$ ); los animales que consumieron una dieta casera obtuvieron los promedios más altos (Tabla 2). Por otro lado, los factores tamaño de la raza y niveles de glucosa, también presentaron diferencias, pero a un nivel menor de significancia ( $\mathrm{p}<0.05)$; en este sentido, los animales de razas grandes presentaron los niveles más altos de glucosa (Tabla 3 ).

Tabla 2. Niveles medios de glucosa según la dieta en perros adultos ( $>7$ años) y con condición corporal mayor o igual a 4 (sobrepeso u obesidad) en la ciudad de Cuenca, Ecuador.

\begin{tabular}{lcc}
\hline Dietas & $\bar{X} \mathrm{mg} \mathrm{dl}^{-1}$ & $\mathrm{EE}^{1}$ \\
\hline Balanceado & $65.15^{\mathrm{a}}$ & 0.82 \\
Casera & $67.66^{\mathrm{b}}$ & 0.71 \\
Balanceado + casera & $65.63^{\mathrm{a}}$ & 0.68 \\
\hline
\end{tabular}

Leyenda: valores con superíndices $a$ difieren significativamente con respecto a $b(\mathrm{p}<0.05)$, según Kramer (1956).

Tabla 3. Niveles medios de glucosa según grupo racial en perros adultos ( $>7$ años) y con condición corporal mayor o igual a 4 (sobrepeso u obesidad) en la ciudad de Cuenca, Ecuador.

\begin{tabular}{lcc}
\hline Grupo racial & $\bar{X} \mathrm{mg} \mathrm{dl}^{-1}$ & $\mathrm{EE}$ \\
\hline Pequeña & $61.98^{\mathrm{a}}$ & 0.66 \\
Mediana & $65.89^{\mathrm{b}}$ & 0.71 \\
Grande & $70.55^{\mathrm{c}}$ & 0.86
\end{tabular}

Leyenda: los valores con superíndices a, b y c, difieren entre si significativamente ( $\mathrm{p}<0.05)$, según Kramer (1956).

\section{DISCUSIÓN}

La hiperglucemia fisiológica es generalmente diagnosticada cuando los niveles de glucosa están entre 120 a $180 \mathrm{mg} \mathrm{dl}^{-1}$, luego de 2 horas después de haber ingerido alimento. Valores sobre $180 \mathrm{mg} \mathrm{dl}^{-1}$, en animales en ayunas, podrían indicar problemas del metabolismo en los carbohidratos y la presencia de diabetes mellitus. Este desequilibrio metabólico puede ser confirmado si además existe presencia de glucosuria y la manifestación de signos clínicos como: poliuria, polidipsia, polifagia y letargia (Bonagura \& Twedt, 2009).

Los niveles normales de glucemia encontrados en el $73.2 \%$ de los caninos en estudio variaron entre 60 a $120 \mathrm{mg} \mathrm{dl}^{-1}$; resultados similares fueron determinados por Paredes (2012) al estudiar en 100 caninos los niveles de glucemia, donde un $72 \%$ tuvieron niveles normales de glucosa en la sangre, con rangos entre 60 a $119 \mathrm{mg} \mathrm{dl}^{-1}$. Otros investigadores como Mesa \& Castillo (2014), al estudiar la prevalencia de diabetes mellitus en perros en un barrio de Nicaragua, calcularon niveles semejantes, entre 61 a $100 \mathrm{mg}$ $\mathrm{dl}^{-1}$ en un $76 \%$ de la población. La frecuencia de animales normoglucémicos es similar en todas las investigaciones revisadas.

En cuanto al porcentaje de animales hipoglucémicos encontrados en esta investigación (26.8\%), comparado con los resultados de un estudio similar realizado por Paredes (2012), en el que no se encontraron animales con niveles de glucemia bajo el rango normal, esta discordancia podría deberse al periodo de ayuno al que fueron sometidos los animales del presente estudio previo a la evaluación. En este aspecto, nuestros resultados son comparables con los de Mesa \& Castillo (2014), quienes encontraron un porcentaje del 20\% de animales hipoglucémicos (niveles de glucosa entre 45 a $60 \mathrm{mg}$

${ }^{1}$ EE: Error estándar 
$\mathrm{dl}^{-1}$ ), estos bajos niveles pudieron deberse a que se realizó un ayuno de al menos 8 horas en los animales en estudio para obtener la muestra.

En nuestro estudio no se encontraron niveles de hiperglucemia que indiquen la presencia de diabetes mellitus, si bien sí se evidenciaron diferentes niveles de glucosa, pero todos dentro del rango normal. Al comparar estos valores con las diferentes categorías de edad, no hubieron correlaciones significativas; por lo tanto, la edad no influyó en los niveles de glucemia, como sugieren otros autores. Sin embargo, Guptill et al. (2003) encontraron el mayor porcentaje de prevalencia en perros con edades comprendidas entre: 5-7 años; 8-10 años y 11-15 años. Paredes (2012) manifestó en su investigación que un $28 \%$ de animales presentaron hiperglucemia, con valores que fluctuaron entre $135 \mathrm{y}^{165 \mathrm{mg} \mathrm{dl}^{-}}$ ${ }^{1}$, si bien estos niveles sobrepasan el rango de $120 \mathrm{mg} \mathrm{dl}^{-1}$, no superan los $180 \mathrm{mg} \mathrm{dl}^{-1}$ que indicarían la presencia de diabetes mellitus. Estos niveles de hiperglucemia encontrados pudieron deberse a una reciente ingesta de alimento, ya que el autor no menciona si, antes de la toma de muestras, hubo un periodo de ayuno de los animales evaluados. Recordemos que el alimento, según su composición química, aporta energía que puede elevar los niveles de glucosa hasta por 2 horas postprandial (Carciofi et al., 2008). Otros autores como Mesa \& Castillo (2014), en perros mayores de 5 años, calcularon un $5 \%$ de animales con niveles de glucosa entre 136 a $180 \mathrm{mg} \mathrm{dl}^{-1}$. En este grupo, los investigadores concluyeron que estos resultados podrían deberse a la mayor cantidad de canes hembras en el estudio y que éstas pudieron estar en la etapa de diestro, donde la presencia de progesterona provoca resistencia a la acción de la insulina. En el mismo estudio, un $4 \%$ de animales presentaron niveles sobre $180 \mathrm{mg} \mathrm{dl}^{-}$ ${ }^{1}$, a pesar de estar en ayunas, presumiendo que estos animales tendrían diabetes. Los niveles altos de glucosa podrían también deberse a una pancreatitis crónica, ocasionada por alteraciones endócrinas, que ocasiona la destrucción de las células beta, responsables de la formación de insulina (Kumar et al., 2014).

La raza es otro factor de riesgo que puede predisponer la aparición de la enfermedad. Al estudiar los niveles de glucosa de los valores normales obtenidos, las razas de perros más grandes (Golden, Pastor Alemán, Pitbull, Labrador y Husky) obtuvieron los valores más altos (70.55 $\left.\mathrm{mg} \mathrm{dl}^{-1}\right)$. En un estudio similar, Davison (2005), demostró que el Samoyedo, Terrier Tibetano y Cairn Terrier tuvieron el riesgo relativo más alto de diabetes. Estas diferencias entre razas sugieren el efecto de un componente genético asociado a la enfermedad. Al realizar estudios en la raza Caniche, Marmor et al. (1982) llegaron a la misma conclusión: existían diferencias estadísticas de riesgo de presencia de DM en esta raza.

En cuanto a la obesidad, evaluada como factor de riesgo para la presencia de DM, aun cuando en el presente estudio todos los animales tenían una condición corporal de sobrepeso u obesidad, estos no presentaron niveles de hiperglucemia. Similares hallazgos fueron evidenciados en un estudio transversal realizado en 117 gatos, en el que no se encontraron diferencias en las concentraciones basales de glucosa en sangre o fructosamina entre gatos con sobrepeso/obesidad y aquellos con peso normal (Clark, 2016). De forma similar, las concentraciones de glucosa en plasma no aumentan necesariamente con obesidad o disminución con pérdida de peso en perros (Mattheuws et al., 1984). A pesar de la resistencia periférica a la insulina, los gatos y perros obesos son capaces de mantener las concentraciones normales de glucosa en el plasma sanguíneo durante largos períodos de tiempo (Clark, 2016).

En la relación entre los factores dieta y niveles de glucosa, los perros que recibieron una alimentación casera presentaron valores más altos dentro del rango normal $67.55 \mathrm{mg} \mathrm{dl}^{-1}$. El almidón, presente como nutriente principal en la dieta de las mascotas, es responsable de alterar e influir en las respuestas postprandiales de insulina y glucosa tanto en perros como en seres humanos (Carciofi et al., 2008). Otro factor dietético que influye en esta respuesta postprandial es la composición de la dieta (Nuttall et al., 1984), por lo general, el alimento que se le proporciona al animal suele consistir en residuos alimenticios en los que se encuentran elementos con alto valor energético.

\section{CONCLUSIONES}

No existe prevalencia de diabetes mellitus en la población de perros estudiados en la ciudad de Cuenca, Ecuador. El 76\% de los animales estudiados presentan niveles normales de glucosa en sangre. El factor genético, como la raza, sí determinó niveles de glucosa diferentes, siendo las razas de mayor tamaño las 
que presentan niveles más altos dentro del rango normal. No se encontraron niveles de hiperglucemia en animales mayores a 7 años que se puedan asociar a la edad, incluso los diferentes rangos de glucosa entre los diversos grupos de edad no fueron significativos. Esto, a pesar de que en investigaciones similares, los perros de mayor edad son los que generalmente presentan una mayor prevalencia a diabetes. La condición de sobrepeso en perros fue un factor que no influenció en la aparición de hiperglucemia. Para avanzar en el diagnóstico de diabetes mellitus sería pertinente hacer pruebas complementarias de glucosuria, fructosamina y glucosa oxidasa, estas pruebas permitirán cotejar los presentes resultados del glucómetro. Este estudio también puede ser complementado con pruebas de biología molecular, para determinar si factores ambientales son los que realmente influyen en esta enfermedad, si es originada por una destrucción autoinmune o por factores ambientales.

\section{REFERENCIAS}

Bonagura DJ, Twedt DC (2009). Kirk's current veterinary therapy XIV. St. Louis, Missouri, USA: Saunders, Elsevier Inc. Disponible en http://www.kestrel.ws/erasmus/docs/ Kirks_Current_Veterinary_Therapy_XIV.pdf,1437 pp

Carciofi AC, Takakura FS, Teshima E, Jeremias JT, Brunetto MA (2008). Effects of six carbohydrate sources on dog diet digestibility and post-prandial glucose and insulin response. Journal of Animal Physiology and Animal Nutrition 92:326-336.

Clark M (2016). Metabolic effects of obesity and its interaction with endocrine diseases. Veterinary Clinics of NA: Small Animal Practice 46(5):797-815

Catchpole B, Ristic JM, Fleeman LM, Davison LJ (2005). Canine diabetes mellitus: Can old dogs teach us new tricks? Diabetologia 48(10):1948-1956

Cunningham JG, Klein BG (2009). Fisiología veterinaria (4 ${ }^{\text {ta }}$ ed.). Barcelona, España:Elsevier. Disponible en http://www.fcv.uagrm.edu.bo/sistemabibliotecario/doc_libros/591\%202647\%20 Fisiolog\%C3\%ADa\%20Veterinaria-Cunningham(4ta\%20Ed)-20100906-104049.pdf, 718 pp

Davison LJ (2005). Canine diabetes mellitus: can old dogs teach us new tricks? Diabetologia 48(10):1948-1956

Fall T, Hamlin HH, Hedhammar A, Kämpe O, Egenvall A (2007). Diabetes mellitus in a population of 180,000 insured dogs: incidence, survival, and breed distribution. Journal of Veterinary Internal Medicine 21(6):1209-1216

Fleeman L, Rand J (2006). Enciclopedia de la nutrición canina. In: Pibot P, Biourge V, Elliot D (Eds.). Diabetes mellitus canina: Estrategia Nutricional. Disponible en http://www.royalcanin.es/ wp-content/uploads/2016/05/Cap-6-Diabetes-Mellitus-canina-Estrategia-nutricional.pdf, 31 pp

Guptill L, Glickman L, Glickman N, Lafayette W, Lafayette W, Lafayette W (2003). Time trends and risk factors for diabetes mellitus in dogs: analysis of veterinary medical data base records (19701999). Veterinary Journal 165(3):240-247

Hardy RM (1988). Diabetes mellitus en el perro y en el gato. Revista oficial de AVEPA, Asociación Veterinaria Española de Especialistas en Pequeños Animales 8(2):71-88. Disponible en https://ddd.uab.cat/pub/clivetpeqani/11307064v8n2/11307064v8n2p71.pdf

Kang M, Kim D, Jeong I, Choi G (2016). Evaluation of four portable blood glucose meters in diabetic and non-diabetic dogs and cats. Veterinary Quarterly 36(1):2-9

Kramer CY (1956). Extension of Multiple Range Tests to Group Means with Unequal Numbers of Replications. Biometrics 12:307

Kumar P, Kumari RR, Kumar M, Kumar S, Chakrabarti A (2014). Current practices and research updates on diabetes mellitus in canine. Veterinary World 7:952-959

Laflamme D (1997). Development and validation of a body condition score system for dogs. Canine Practice 22:10-15

Mattheuws D, Rottiers R, Baeyens D (1984). Glucose tolerance and insulin response in obese dogs. 
Journal of the American Animal Hospital Association 20:287-293

Marmor M, Willeber P, Glickman L, Priester W, Cypess R, Hurvitz A (1982). Epizootiologic patterns of diabetes mellitus in dogs. American Journal of Veterinary Research 65(8):467-470

Mesa D, Castillo A (2014). Prevalencia de diabetes mellitus en caninos con edad mayor o igual a 5 años del barrio Juan Alberto Blandón del municipio de Estelí utilizando como método diagnóstico el Glucómetro ACON On Callß en el periodo comprendido de Agosto a Septiembre 2013. Tesis de pregrado, Universidad Nacional Autonoma de Nicaragua. Disponible en http://riul.unanleon.edu.ni:8080/jspui/bitstream/123456789/3307/1/228063.pdf, 36 pp

Nuttall FQ, Mooradian AD, Gannon MC, Billington C, Krezowski P (1984). Effect of protein ingestion on the glucose and insulin response to a standardized oral glucose load, Diabetes Care 7(5):465-470

Paredes J (2012). Frecuencia de hiperglucemia subclinica o asintomatica en caninos mayores de siete años de un centro de rescate. Revista de la Universidad Científica del Sur 9(21):79-87

Rand JS, Fleeman LM, Farrow HA, Appleton DJ (2004). Canine and feline diabetes mellitus: nature or nurture? Journal of Nutrition 134(8 Suppl):2072S-2080S

SAS (2013). SAS User's guide: Statistics, version 9.3. The SAS Institute INC, Cary NC, USA

Wiedmeyer CE, Declue AE (2011). Glucose monitoring in diabetic dogs and cats: Adapting new technology for home and hospital care. Clinics in Laboratory Medicine 31(1):41-50 